\title{
ASYMPTOTIC ANALYSIS OF AMERICAN CALL OPTIONS
}

\author{
GHADA ALOBAIDI and ROLAND MALLIER
}

(Received 7 August 2000)

\begin{abstract}
American call options are financial derivatives that give the holder the right but not the obligation to buy an underlying security at a pre-determined price. They differ from European options in that they may be exercised at any time prior to their expiration, rather than only at expiration. Their value is described by the Black-Scholes PDE together with a constraint that arises from the possibility of early exercise. This leads to a free boundary problem for the optimal exercise boundary, which determines whether or not it is beneficial for the holder to exercise the option prior to expiration. However, an exact solution cannot be found, and therefore by using asymptotic techniques employed in the study of boundary layers in fluid mechanics, we find an asymptotic expression for the location of the optimal exercise boundary and the value of the option near to expiration.
\end{abstract}

2000 Mathematics Subject Classification. 91B28, 41A58.

1. Introduction. Recently, the financial markets have seen an explosion of "derivative products" such as options. An option is a contract that allows the holder to buy or sell a financial asset at a fixed price in the future. Options need not be exercised, the holder of the option will use it only if this is convenient. A call is an option to buy an asset and a put is an option to sell it. An option contract specifies the exercise price and the expiration date of the contract.

Such options exist on many assets (known as underlyers). Options are a special type of derivative security because their value is derived from the value of some underlying security. Most options can be grouped into either of two categories: European options which can be exercised only on their expiration date, and American options which can be exercised on or before their expiration date. In practice, most options are American. American options are much harder to deal with than European ones. The problem is that it may be optimal to use (exercise) the option before the final expiry date. This optimal exercise policy will affect the value of the option, and the exercise policy needs to be known when solving the PDE. Holders of American options have this choice of when to exercise the options.

The main problem of options is how they should be priced in equilibrium with the price and characteristics of the underlying asset. This problem was solved by Black and Scholes [1]. Some financial institutions make money by selling large number of options. They make money on some and lose money on others. This can only happen if they are selling the options at a correct price. Options can be used as a speculative medium with small, or relatively small, risk and with unlimited possible profit.

The growth in the availability of financial derivatives has led to (and in part been driven by) the development of mathematical models which are used to value these options, with the Black-Scholes model being the best known of these. 
In this paper, a model for pricing American call options on dividend paying assets is presented, so we will concentrate on American call option but there are also barrier options, Asian options, and so forth. For American call options on nondividend paying assets, early exercise is never optimal, and the early exercise premium is zero. For options on dividend-paying stocks, early exercise may be optimal for some stockprice paths, making the early exercise premium positive. For the special case of one dividend payment during the life of an option, an analytical solution is available, due to Roll, Geske, and Whaley. A first formulation of an analytical call price with dividends was given by Roll [6]. This had some errors that were partially corrected in Geske [3], before Whaley [7] gave a final, correct formula. Geske and Johnson [4] used the series of Bermudan option prices to approximate the price of American options.

Valuation of American options is more complicated, since at each time we have to determine not only the option value, but also, whether or not it should be exercised and this leads to a free boundary problem, with the boundary lying between the regions, where early exercise is beneficial and where it is not. The presence of this free boundary makes the mathematics of American options more complicated than their European counterparts, and much of the work done to date on American options has been numerical.

To value American options, the idea is that we should look for a function $C(S, t)$ that satisfies the Black-Scholes equation in the region of the $(S, t)$-plane, where the option should not be exercised and provide additional boundary conditions along the region where the option should be exercised. To arrive at this region is to impose the additional conditions on option prices that should hold in the case of American style options. As long as exercise is not optimal, the payoff condition is $C(S, T)=$ $\max (S-E, 0)$ but because the American option can be exercised at any time, we always have

$$
C(S, t) \geq \max (S-E, 0) .
$$

In this case if $S>E$, then the option is in the money. If $S<E$, the option is out of the money. If $S=E$, the option is at the money. The converse is true for the put options. The rest of the paper is organized as follows. In Section 2, we describe the analysis of the American call option using the Black-Scholes model. This analysis is based on arbitrage arguments. Also, we discuss the optimal exercise boundary $x_{f}(\tau)$, where $x_{f}(\tau)$ is not known, therefore, the problem of determining the option price is then a free boundary problem. In particular, we will discuss the optimal exercise price for an American call on a dividend-paying asset at times near expiry. We used asymptotic expansions to find the free boundary. American options have been considered previously by Jacka [5] from the perspective of optimal stopping-time problems. Section 3 presents graphical results of the free boundary and the price of the call option. Section 4 contains a summary for analysis and a brief discussion.

2. Black-Scholes PDE for American options. In their monograph "Option Pricing" (see [8, pages 110-119]), Wilmott, Dewynne, and Howison, lay the foundation for an asymptotic analysis of American call options near to expiration. However, they only take the analysis to first order in order to verify their numerical results, and do not 
pursue it further. In this section, we take their analysis to higher orders. Our $x_{1}$ and $\kappa_{0}$ were the end result of their analysis. The foundation of our analysis therefore follows the one in "Option Pricing" very closely.

From "Option Pricing," an American call option on an underlying that pays a continuous dividend obeys the following (Black-Scholes) PDE:

$$
\frac{\partial C}{\partial t}+\frac{1}{2} \sigma^{2} S^{2} \frac{\partial^{2} C}{\partial S^{2}}+\left(r-D_{0}\right) S \frac{\partial C}{\partial S}-r C=0
$$

where

$t$ : time

$\sigma$ : volatility of the underlying asset

$S$ : price of underlying stock

$C$ : price of call option

$r$ : interest rate

$D_{0}$ : dividend yield.

Because this option can be exercised at any time, we also have the constraint

$$
C(S, t) \geq \max (S-E, 0) .
$$

To facilitate our analysis, we make the following change of variables:

$$
S=E e^{x}, \quad t=T-\frac{\tau}{(1 / 2) \sigma^{2}}, \quad C(S, t)=S-E+E c(x, \tau) .
$$

After transformation we get

$$
\frac{\partial c}{\partial \tau}=\frac{\partial^{2} c}{\partial x^{2}}+\left(k_{2}-1\right) \frac{\partial c}{\partial x}-k_{1} c+f(x)
$$

for $-\infty<x<\infty$ and $\tau>0$, where

$$
f(x)=\left(k_{2}-k_{1}\right) e^{x}+k_{1}=k_{1}\left(1-e^{x-x_{0}}\right), \quad x_{0}=\log \left(\frac{k_{1}}{k_{1}-k_{2}}\right) .
$$

The two parameters $k_{1}$ and $k_{2}$ are given by

$$
k_{1}=\frac{r}{(1 / 2) \sigma^{2}}, \quad k_{2}=\frac{r-D_{0}}{(1 / 2) \sigma^{2}}, \quad k_{1}>k_{2}>0 .
$$

We must solve these equations together with the boundary condition that

$$
c(x, 0)=\max \left(1-e^{x}, 0\right)= \begin{cases}1-e^{x}, & x<0, \\ 0, & x \geq 0,\end{cases}
$$

and the constraint on $c$ that

$$
c(x, \tau) \geq \max \left(1-e^{x}, 0\right) .
$$

Because of this, there will be a free boundary, which we suppose to be located at $x=x_{f}(\tau)$, where

$$
c\left(x_{f}(\tau), \tau\right)=\frac{\partial c}{\partial x}\left(x_{f}(\tau), \tau\right)=0
$$


that is to say, both $c$ and $\partial c / \partial x$ vanish at the free boundary. The location of the free boundary is given by $x_{f}(\tau)$, where $x_{f}$ is an unknown function. The purpose of this study is to find an asymptotic expression for $x_{f}$. At expiration, we know that

$$
x_{f}(0)=x_{0}
$$

where $x_{0}$ is defined in (2.5) and $f\left(x_{0}\right)=0$. Near to expiration, we expand $x_{f}$ in $\tau$

$$
x_{f}(\tau)=x_{0}+x_{1} \tau^{1 / 2}+x_{2} \tau+x_{3} \tau^{3 / 2}+x_{4} \tau^{2}+x_{5} \tau^{5 / 2}+\cdots
$$

We perform a local analysis in the vicinity of $x=x_{0}$ and $\tau=0$, and introduce the rescaled coordinates,

$$
\begin{aligned}
& x-x_{0}=v X, \quad \tau=\mu \xi, \quad c(x, \tau)=\varepsilon \gamma(X, \xi), \\
& f(x) \sim-k_{1}\left(v X+\frac{\nu^{2} X^{2}}{2 !}+\frac{\nu^{3} X^{3}}{3 !}+\frac{\nu^{4} X^{4}}{4 !}+\cdots\right),
\end{aligned}
$$

where $v \ll 1, \mu \ll 1$, and $\varepsilon \ll 1$ are small parameters. With these rescaled variables, the PDE becomes

$$
\begin{aligned}
\varepsilon \mu^{-1} \frac{\partial \gamma}{\partial \xi}= & \varepsilon \nu^{-2} \frac{\partial^{2} \gamma}{\partial X^{2}}+\varepsilon \nu^{-1}\left(k_{2}-1\right) \frac{\partial \gamma}{\partial X}-\varepsilon k_{1} \gamma \\
& -k_{1}\left(v X+\frac{v^{2} X^{2}}{2 !}+\frac{\nu^{3} X^{3}}{3 !}+\frac{v^{4} X^{4}}{4 !}+\cdots\right),
\end{aligned}
$$

with $\gamma(X, \xi=0)=0$ at expiration. If we consider the balance of terms in (2.13), to leading order we must have

$$
\varepsilon \mu^{-1} \frac{\partial \gamma}{\partial \xi} \sim \varepsilon v^{-2} \frac{\partial^{2} \gamma}{\partial X^{2}}-v k_{1} X
$$

This gives us a relationship between $\varepsilon, \mu$, and $\nu$, since we require that each term in (2.14) be of the same order of magnitude. Therefore we must have $\mu=v^{2}$ and $\varepsilon=v^{3}$, so that (2.12) becomes

$$
\begin{gathered}
x-x_{0}=v X, \quad \tau=v^{2} \xi, \quad c(x, \tau)=v^{3} \gamma(X, \xi), \\
f(x) \sim-k_{1}\left(v X+\frac{v^{2} X^{2}}{2 !}+\frac{v^{3} X^{3}}{3 !}+\frac{v^{4} X^{4}}{4 !}+\cdots\right),
\end{gathered}
$$

and (2.13) becomes

$$
\frac{\partial \gamma}{\partial \xi}=\frac{\partial^{2} \gamma}{\partial X^{2}}+v\left(k_{2}-1\right) \frac{\partial \gamma}{\partial X}-v^{2} k_{1} \gamma-k_{1}\left(X+\frac{v X^{2}}{2 !}+\frac{v^{2} X^{3}}{3 !}+\frac{v^{3} X^{4}}{4 !}+\cdots\right) .
$$

Next, we shall expand $\gamma$ as a series in $v$,

$$
\gamma \sim \gamma_{0}+v \gamma_{1}+v^{2} \gamma_{2}+\cdots .
$$


Substituting this expansion into the governing equation (2.16) yields at successive powers of $v$

$$
\begin{aligned}
& \frac{\partial \gamma_{0}}{\partial \xi}=\frac{\partial^{2} \gamma_{0}}{\partial X^{2}}-k_{1} X \\
& \frac{\partial \gamma_{1}}{\partial \xi}=\frac{\partial^{2} \gamma_{1}}{\partial X^{2}}+\left(k_{2}-1\right) \frac{\partial \gamma_{0}}{\partial X}-\frac{k_{1} X^{2}}{2 !}, \\
& \frac{\partial \gamma_{2}}{\partial \xi}=\frac{\partial^{2} \gamma_{2}}{\partial X^{2}}+\left(k_{2}-1\right) \frac{\partial \gamma_{1}}{\partial X}-k_{1} \gamma_{0}-\frac{k_{1} X^{3}}{3 !}, \\
& \frac{\partial \gamma_{3}}{\partial \xi}=\frac{\partial^{2} \gamma_{3}}{\partial X^{2}}+\left(k_{2}-1\right) \frac{\partial \gamma_{2}}{\partial X}-k_{1} \gamma_{1}-\frac{k_{1} X^{4}}{4 !}
\end{aligned}
$$

subject to the condition that at expiration

$$
\gamma_{0}(X, \xi=0)=\gamma_{1}(X, \xi=0)=\gamma_{2}(X, \xi=0)=\cdots=0 .
$$

Condition (2.9) on the free boundary at $x=x_{f}(\tau)$, where both $c$ and $\partial c / \partial x$ vanish, must also be tackled. We can also rewrite the expansion of $x_{f}$ near expiration (2.11) as follows:

$$
x_{f}(\tau)=x_{0}+v x_{1} \xi^{1 / 2}+v^{2} x_{2} \xi+v^{3} x_{3} \xi^{3 / 2}+\cdots .
$$

Thus the free boundary is located at

$$
X_{f}(\xi)=v^{-1}\left(x_{f}(\tau)-x_{0}\right)=x_{1} \xi^{1 / 2}+v x_{2} \xi+v^{2} x_{3} \xi^{3 / 2}+\cdots,
$$

and the boundary condition that $c$ vanish at the free boundary becomes

$$
\gamma_{0}\left(X_{f}(\xi), \xi\right)+v \gamma_{1}\left(X_{f}(\xi), \xi\right)+v^{2} \gamma_{2}\left(X_{f}(\xi), \xi\right)+\cdots=0 .
$$

Similarly, the boundary condition that $\partial c / \partial x$ vanish becomes

$$
\gamma_{0 X}\left(X_{f}(\xi), \xi\right)+v \gamma_{1 X}\left(X_{f}(\xi), \xi\right)+v^{2} \gamma_{2 X}\left(X_{f}(\xi), \xi\right)+\cdots=0 .
$$

At leading order $O\left(v^{0}\right)$, we have from (2.18)

$$
\frac{\partial \gamma_{0}}{\partial \xi}=\frac{\partial^{2} \gamma_{0}}{\partial X^{2}}-k_{1} X
$$

while substituting the expansion (2.21) into the conditions on $c$ at the free boundary (2.22), (2.23) yields at leading order

$$
\gamma_{0}\left(x_{1} \xi^{1 / 2}, \xi\right)=\gamma_{0 X}\left(x_{1} \xi^{1 / 2}, \xi\right)=0 .
$$

Since (2.24) is the diffusion equation together with a nonhomogeneous term, this suggests introducing the similarity variable

$$
\eta=X \xi^{-1 / 2}
$$

Accordingly, we write $\gamma_{0}=\xi^{3 / 2} \kappa_{0}(\eta)$, and substituting this into (2.24) gives

$$
\frac{3}{2 \kappa_{0}}-\frac{1}{2 \eta \kappa_{0 \eta}}=\kappa_{0 \eta \eta}-k_{1} \eta
$$


together with the conditions at the free boundary

$$
\kappa_{0}\left(x_{1}\right)=\kappa_{0 \eta}\left(x_{1}\right)=0 .
$$

This has the solution

$$
\kappa_{0}(\eta)=-k_{1} \eta+C_{1}^{(0)}\left(\eta^{3}+6 \eta\right)+C_{2}^{(0)}\left(e^{-\eta^{2} / 4}\left(\eta^{2}+4\right)+\frac{\sqrt{\pi}}{2}\left(\eta^{3}+6 \eta\right) \operatorname{erfc}\left(-\frac{\eta}{2}\right)\right) .
$$

We also need to apply condition (2.19) that $\gamma_{0}$ vanish at expiration. Since we set $\eta=X \xi^{-1 / 2}$, and $X<0$, the limit $\xi \rightarrow 0$ corresponds to the limit $\eta \rightarrow-\infty$. Taking this limit, we get

$$
\begin{gathered}
\kappa_{0}(\eta) \longrightarrow-k_{1} \eta+C_{1}^{(0)}\left(\eta^{3}+6 \eta\right), \\
\gamma_{0}=\xi^{3 / 2} \kappa_{0}(\eta) \longrightarrow-k_{1} \xi X+C_{1}^{(0)}\left(X^{3}+6 \xi X\right) \longrightarrow C_{1}^{(0)} X^{3},
\end{gathered}
$$

and thus the condition that $\gamma_{0}$ vanishes in this limit tells us that $C_{1}^{(0)}=0$, and the solution (2.29) becomes

$$
\kappa_{0}(\eta)=-k_{1} \eta+C_{2}^{(0)}\left(e^{-\eta^{2} / 4}\left(\eta^{2}+4\right)+\frac{\sqrt{\pi}}{2}\left(\eta^{3}+6 \eta\right) \operatorname{erfc}\left(-\frac{\eta}{2}\right)\right) .
$$

The condition (2.28) at the free boundary enables us to find $x_{1}$ and $C_{2}^{(0)}$, where $x_{1}$ is given implicitly by the equation

$$
\left(4-2 x_{1}^{2}\right)=\sqrt{\pi} x_{1}^{3} e^{x_{1}^{2} / 4} \operatorname{erfc}\left(-\frac{x_{1}}{2}\right) .
$$

Numerically, we find

$$
x_{1}=0.9034465979, \quad C_{2}^{(0)}=0.07536083707 k_{1} .
$$

Thus $C_{2}^{(0)}$ is proportional to the constant $k_{1} . x_{1}$ and $\kappa_{0}$ were found by Wilmott et al. [8], however, their analysis stopped there, whilst we shall proceed to higher orders.

At the next order $O(v)$, we get an equation for $\gamma_{1}$,

$$
\frac{\partial \gamma_{1}}{\partial \xi}-\frac{\partial^{2} \gamma_{1}}{\partial X^{2}}=\left(k_{2}-1\right) \frac{\partial \gamma_{0}}{\partial X}-\frac{k_{1} X^{2}}{2 !} .
$$

Again we make use of the similarity variable (2.26) and write $\gamma_{1}=\xi^{2} \kappa_{1}(\eta)$. Substituting this into (2.34) yields,

$$
2 \kappa_{1}-\frac{1}{2} \eta \kappa_{1 \eta}-\kappa_{1 \eta \eta}=\left(k_{2}-1\right) \kappa_{0 \eta}-\frac{k_{1}}{2} \eta^{2},
$$

which has the solution

$$
\begin{aligned}
\kappa_{1}(\eta)= & -\frac{1}{2} k_{1}\left(k_{2}+\eta^{2}\right)+C_{1}^{(1)}\left(\eta^{4}+12 \eta^{2}+12\right) \\
& +C_{2}^{(0)}\left(1-k_{2}\right)\left(e^{-\eta^{2} / 4}\left(-\frac{1}{2}+\frac{\eta^{2}}{4}\right) \eta+\sqrt{\pi}\left(\frac{\eta^{4}}{8}-\frac{3}{2}\right) \operatorname{erfc}\left(-\frac{\eta}{2}\right)\right) \\
& +C_{2}^{(1)}\left(e^{-\eta^{2} / 4}\left(20 \eta+2 \eta^{3}\right)+\sqrt{\pi}\left(\eta^{4}+12 \eta^{2}+12\right) \operatorname{erfc}\left(-\frac{\eta}{2}\right)\right),
\end{aligned}
$$

where again $C_{1}^{(1)}$ must vanish because of the condition at expiry. 
The boundary conditions at the free boundary are

$$
\kappa_{1}\left(x_{1}\right)=0, \quad \kappa_{1 \eta}\left(x_{1}\right)+x_{2} \kappa_{0 \eta \eta}\left(x_{1}\right)=0
$$

Applying these boundary conditions to $\kappa_{0}$ and $\kappa_{1}$ enables us to find $x_{2}$ and $C_{2}^{(1)}$,

$$
x_{2}=-\frac{x_{1}^{2} k_{2}}{x_{1}^{2}+2}, \quad C_{2}^{(1)}=\frac{x_{1}^{3} e^{x_{1}^{2} / 4} k_{1}\left(x_{1}^{2}+2+x_{1}^{2} k_{2}\right)}{96\left(x_{1}^{2}+2\right)} .
$$

Using the value of $x_{1}$ found earlier, these become

$$
x_{2}=-0.2898271391 k_{2}, \quad C_{2}^{(1)}=0.009420104644 k_{1}+0.002730201979 k_{1} k_{2} .
$$

At the next order $\mathcal{O}\left(v^{2}\right)$, we find an equation for $\gamma_{2}$,

$$
\frac{\partial \gamma_{2}}{\partial \xi}-\frac{\partial^{2} \gamma_{2}}{\partial X^{2}}=\left(k_{2}-1\right) \frac{\partial \gamma_{1}}{\partial X}-\frac{k_{1} X^{3}}{3 !}-k_{1} \gamma_{0}
$$

Using $\gamma_{2}=\xi^{5 / 2} \kappa_{2}(\eta)$ in (2.40), we get

$$
\frac{5}{2} \kappa_{2}-\frac{1}{2 \eta} \kappa_{2 \eta}-\kappa_{2 \eta \eta}=\left(k_{2}-1\right) \kappa_{1 \eta}-\frac{1}{6} k_{1} \eta^{3}-k_{1} \kappa_{0} .
$$

The solution $\kappa_{2}$ is given in the appendix.

The boundary conditions on $\kappa_{2}$ at the free boundary are

$$
\begin{gathered}
\frac{x_{2}^{2}}{2} \kappa_{0 \eta \eta}\left(x_{1}\right)+\kappa_{2}\left(x_{1}\right)=0 \\
\frac{1}{2} x_{2}^{2} \kappa_{0 \eta \eta \eta}\left(x_{1}\right)+x_{3} \kappa_{0 \eta \eta}\left(x_{1}\right)+\kappa_{2 \eta}\left(x_{1}\right)+x_{2} \kappa_{1 \eta \eta}\left(x_{1}\right)=0,
\end{gathered}
$$

which enable us to find $x_{3}$ and $C_{2}^{(2)}$. Numerically, we find

$$
\begin{aligned}
x_{3}= & 0.08352705033 k_{2}-0.1670541006 k_{1} \\
& -0.01960251625+0.0965932214 k_{2}^{2}, \\
C_{2}^{(2)}= & 0.0008901468022 k_{1} k_{2}+0.001931411733 k_{1} \\
& -0.0001421724195 k_{1}^{2}-0.0004594870885 k_{1} k_{2}^{2} .
\end{aligned}
$$

At $\mathcal{O}\left(v^{3}\right), \gamma_{3}$ obeys the equation

$$
\frac{\partial \gamma_{3}}{\partial \xi}-\frac{\partial^{3} \gamma_{3}}{\partial X^{2}}=\left(k_{2}-1\right) \frac{\partial \gamma_{2}}{\partial X}-\frac{k_{1} X^{4}}{4 !}-k_{1} \gamma_{1}
$$

Writing $\gamma_{3}=\xi^{3} \kappa_{3}(\eta)$, we get

$$
3 \kappa_{3}-\frac{1}{2} \eta \kappa_{3 \eta}-\kappa_{3 \eta \eta}=\left(k_{2}-1\right) \kappa_{2 \eta}-\frac{1}{24} k_{1} \eta^{4}-k_{1} \kappa_{1}(\eta) .
$$

The solution $\kappa_{3}$ is given in the appendix. The boundary conditions on $\kappa_{3}$ at the free 
boundary are

$$
\begin{aligned}
\frac{1}{6} x_{2}^{3} \kappa_{0 \eta \eta \eta}\left(x_{1}\right) & +x_{2} x_{3} \kappa_{0 \eta \eta}\left(x_{1}\right)+\frac{1}{2} x_{2}^{2} \kappa_{1 \eta \eta}\left(x_{1}\right) \\
& +x_{3} \kappa_{1 \eta}\left(x_{1}\right)+x_{1} \kappa_{3}\left(x_{1}\right)+x_{2} \kappa_{2 \eta}\left(x_{1}\right)=0, \\
\frac{1}{6} x_{2}^{3} \kappa_{0 \eta \eta \eta \eta}\left(x_{1}\right) & +x_{2} x_{3} \kappa_{0 \eta \eta \eta}\left(x_{1}\right)+x_{4} \kappa_{0 \eta \eta}\left(x_{1}\right)+\frac{1}{2} x_{1} x_{2}^{2} \kappa_{1 \eta \eta \eta}\left(x_{1}\right) \\
& +x_{3} \kappa_{1 \eta \eta}\left(x_{1}\right)+x_{2} \kappa_{2 \eta \eta}\left(x_{1}\right)+\kappa_{3 \eta}\left(x_{1}\right)=0 .
\end{aligned}
$$

Using these boundary conditions, we can find $x_{4}$ and $C_{2}^{(3)}$,

$$
\begin{aligned}
x_{4}= & 0.004173449415 k_{2}-0.03134069092 k_{2}^{2} \\
& +0.002104860402 k_{2}^{3}+0.06268138183 k_{1} k_{2}, \\
C_{2}^{(3)}= & 0.0001887470540 k_{1} k_{2}-0.00004739080649 k_{1}^{2} \\
& +0.0003298004233 k_{1}-0.0001854489478 k_{1} k_{2}^{2} \\
& +0.00006457317010 k_{1} k_{2}^{2}+0.0000553581598 k_{1} k_{2}^{3} .
\end{aligned}
$$

Following the same procedure at $\mathcal{O}\left(v^{4}\right)$ and after applying the boundary conditions, the value of $x_{5}$ and $C_{4}^{(2)}$ can be written as

$$
\begin{aligned}
x_{5}= & -0.003558807857 k_{2}+0.007117615715 k_{1}+0.01940343468 k_{1}^{2} \\
& +0.005720713541 k_{2}^{2}-0.01940393468 k_{1} k_{2}-0.003010435594 k_{1} k_{2}^{2} \\
& -0.0001404406593 k_{2}^{4}+0.0007732477173+0.001505467797 k_{2}^{3}, \\
C_{2}^{(4)}= & 0.00003122771752 k_{1} k_{2}-0.000009566207702 k_{1}^{2}-0.0000006665142604 k_{1}^{3} \\
& -0.00004508455720 k_{1} k_{2}^{2}+0.00002372836074 k_{1}^{2} k_{2}-0.00001481303829 k_{1}^{2} k_{2}^{2} \\
& -0.000005222446464 k_{1} k_{2}^{4}+0.00004808832947 k_{1}+0.00002717729053 k_{1} k_{2}^{2} .
\end{aligned}
$$

Thus we have an asymptotic expression (2.11) for the location of the free boundary $x_{f}(\tau)$, with the coefficients $x_{0}, \ldots, x_{5}$ given by (2.5), (2.33), (2.38), (2.43), (2.47), and (2.48). We also have a local expression for the value $c(x, \tau)$ of the option when we are both near to expiry and near to the optimal exercise boundary. This is given by (2.15) and (2.17) together with the expressions for $\gamma_{0}, \ldots, \gamma_{3}$ contained in the text.

3. Graphics. In Figures 2.1 and 2.2 we plot the location of the free boundary for several values of $r(0.102,0.104,0.108$, and 0.110$)$ and of the dividend yield $D_{0}(0.02$, 0.021 , and 0.025$)$. The shape of all the curves appears to be very similar. Figure 2.3 shows the solution of the price option $c(X, \tau)$. The solution increases as long as we move away from the free boundary. Figures 2.1 and 2.2 were produced by including terms up to $x_{6}$ and Figure 2.3 by including terms up to $\gamma_{5}$. 


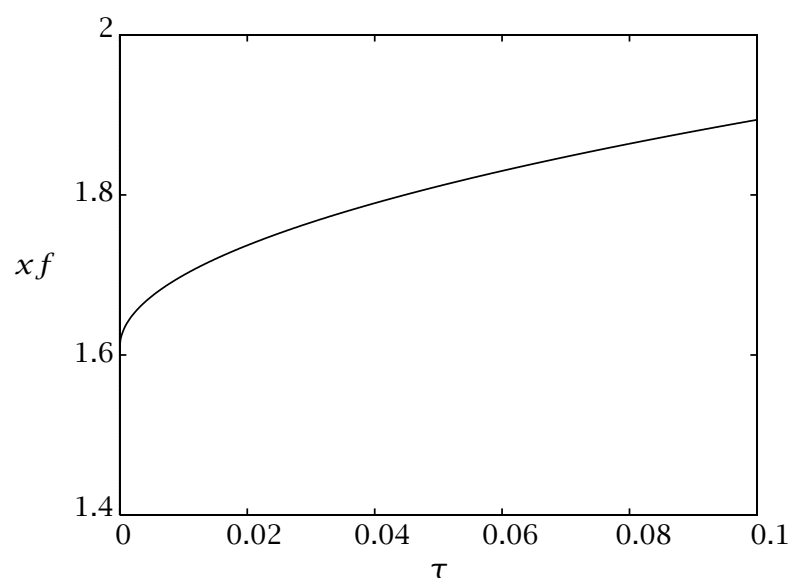

FIGURE 2.1. Location of the free boundary for $r=0.1, D_{0}=0.02$.

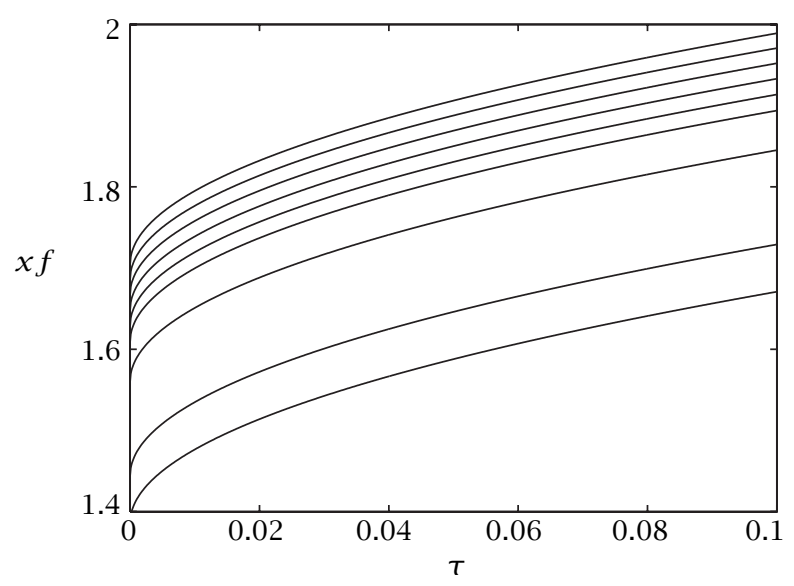

FIGURE 2.2. Location of the free boundary for several values of $r$ and $D_{0}$.

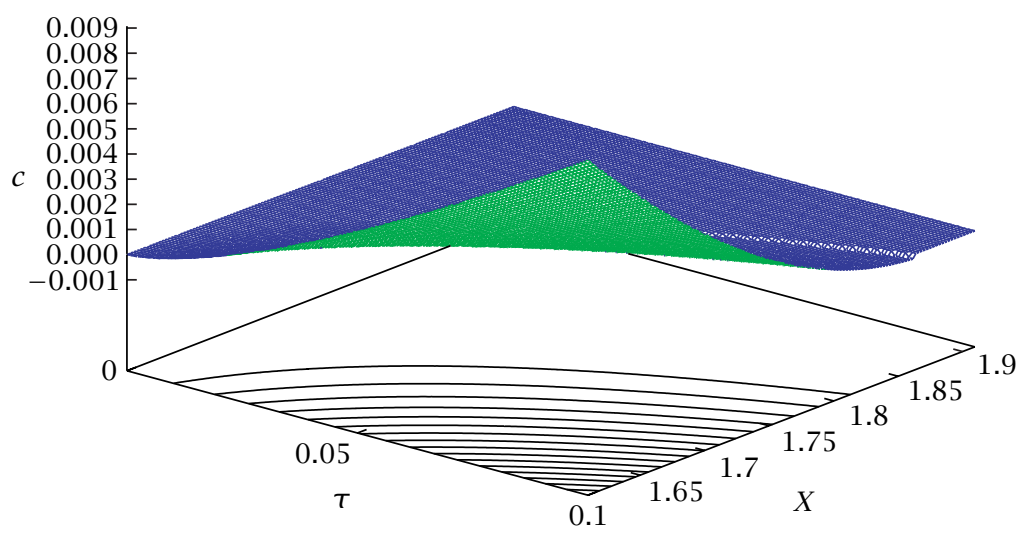

FIGURE 2.3. Solution of the price option $c(X, \tau)$. 
4. Summary and conclusions. In the previous sections, we have presented an asymptotic analysis of the valuation of an American call option on a dividend-paying asset, using as a starting point the Black-Scholes model, which expresses the price of a call option as a function of the underlying asset price, exercise price, the time to expiration, the interest rate, and the volatility of the underlying asset price.

The Black-Scholes model applies to both European and American options as long as no dividends are paid. When dividends are paid, the possibility of early exercise exists to obtain the dividend payment for a call option. (Cox et al. [2] developed some arbitrage conditions for call option.)

The aim of this paper was to use the techniques outlined by Wilmott et al. [8] to solve the free boundary problem arising from early exercise. Using asymptotic techniques, we obtained a series solution for the location of this free boundary near to expiry, and this solution is plotted in Figures 2.1 and 2.2 for several values of $r$, the risk free rate, and $D_{0}$, the dividend yield on the underlying. Using similarity solutions, we were also able to solve a series of partial differential equations to find a local solution for the value $c(x, \tau)$ of the option when we are both near to expiration and near to the optimal exercise boundary and a sample solution was plotted in Figure 2.3. Wilmott et al. [8] had begun this analysis, but stopped at first order, whereas our analysis is pursued to higher orders. This analysis allows for valuation of American call options near to expiry at much lower computational cost than numerical solution of the full problem, and our solution could probably even be programmed into a financial calculator, allowing traders to obtain reasonable valuations quickly.

\section{Appendix}

Details of the analysis. The solution to (2.41) is

$$
\begin{aligned}
\kappa_{2}(\eta)=\frac{1}{2} k_{1}^{2} \eta & -\frac{1}{2} k_{2} k_{1} \eta-\frac{1}{6} k_{1} \eta^{3}+C_{1}^{(2)} \eta\left(\eta^{4}+20 \eta^{2}+60\right) \\
+( & \frac{96}{5} k_{2} C_{2}^{(1)}-\frac{6}{5} k_{2} C_{2}^{(0)}-\frac{96}{5} C_{2}^{(1)}+\frac{3}{5} C_{2}^{(0)}+64 C_{2}^{(2)}-\frac{12}{5} k_{1} C_{2}^{(0)} \\
& +\frac{3}{5} k_{2}^{2} C_{2}^{(0)}-\frac{1}{10} \eta^{2} k_{2}^{2} C_{2}^{(0)}+\frac{4}{5} k_{2} C_{2}^{(1)} \eta^{2}-\frac{1}{10} k_{1} \eta^{2} C_{2}^{(0)} \\
& +\frac{3}{5} k_{2}^{2} C_{2}^{(0)}-\frac{1}{10} \eta^{2} k_{2}^{2} C_{2}^{(0)}+\frac{4}{5} k_{2} C_{2}^{(1)} \eta^{2}-\frac{1}{10} k_{1} \eta^{2} C_{2}^{(0)} \\
& +\frac{1}{5} \eta^{2} k_{2} C_{2}^{(0)}-\frac{4}{5} C_{2}^{(1)} \eta^{2}+36 C_{2}^{(2)} \eta^{2}-\frac{1}{10} \eta^{2} C_{2}^{(0)}+\frac{2}{5} \eta^{4} C_{2}^{(1)} \\
& +\frac{1}{20} \eta^{4} C_{2}^{(0)}-\frac{1}{10} \eta^{4} k_{2} C_{2}^{(0)}+2 C_{2}^{(2)} \eta^{4}-\frac{2}{5} \eta^{4} k_{2} C_{2}^{(1)} \\
& \left.+\frac{1}{20} \eta^{4} k_{2}^{2} C_{2}^{(0)}+\frac{1}{20} \eta^{4} k_{1} C_{2}^{(0)}\right) e^{\left(-1 / 4 \eta^{2}\right)}
\end{aligned}
$$




$$
\begin{aligned}
+\sqrt{\pi}( & -\frac{3}{2} k_{1} C_{2}^{(0)} \eta+12 k_{2} C_{2}^{(1)} \eta-12 C_{2}^{(1)} \eta+\frac{1}{5} \eta^{5} C_{2}^{(1)} \\
& +\frac{1}{40} \eta^{5} k_{2}^{2} C_{2}^{(0)}+\frac{1}{40} \eta^{5} C_{2}^{(0)}-\frac{1}{5} \eta^{5} k_{2} C_{2}^{(1)}-\frac{1}{20} \eta^{5} k_{2} C_{2}^{(0)} \\
& \left.+\frac{1}{40} \eta^{5} k_{1} C_{2}^{(0)}+C_{2}^{(2)} \eta\left(\eta^{4}+20 \eta^{2}+60\right)\right) \operatorname{erfc}\left(-\frac{1}{2} \eta\right)
\end{aligned}
$$

The condition at expiration tells us that $C_{1}^{(2)}=0$.

The solution to (2.45) is

$$
\begin{aligned}
\kappa_{3}(\eta)= & \frac{1}{3} k_{2} k_{1}^{2}-\frac{1}{6} k_{1} k_{2}^{2}-\frac{1}{4} k_{1}^{2} k_{1} \eta^{2}+\frac{1}{4} k_{1}^{2} \eta^{2}-\frac{1}{24} k_{1} \eta^{4} \\
+ & C_{1}^{(3)}\left(\eta^{6}+30 \eta^{4}+180 \eta^{2}+120\right) \\
+ & \frac{1}{20} \eta^{3} k_{2} C_{2}^{(0)}-\frac{1}{60} \eta^{3} C_{2}^{(0)}+\frac{1}{10} C_{2}^{(0)} \eta+\frac{4}{5} C_{2}^{(1)} \eta \\
& -\frac{2}{15} C_{2}^{(1)} \eta^{3}-56 C_{2}^{(2)} \eta-\frac{2}{3} C_{2}^{(2)} \eta^{3}+56 C_{2}^{(3)} \eta^{3} \\
& +264 C_{2}^{(3)} \eta-\frac{3}{10} k_{2} C_{2}^{(0)} \eta-\frac{1}{10} \eta k_{2}^{3} C_{2}^{(0)}-\frac{2}{15} k_{1} C_{2}^{(1)} \eta^{3} \\
& -\frac{56}{5} k_{1} C_{2}^{(1)} \eta-\frac{1}{20} \eta^{3} k_{2}^{2} C_{2}^{(0)}-\frac{1}{30} \eta^{3} k_{1} C_{2}^{(0)}+\frac{1}{5} k_{1} \eta C_{2}^{(0)} \\
& +\frac{4}{15} \eta^{3} k_{2} C_{2}^{(1)}+56 k_{2} C_{2}^{(2)} \eta-\frac{1}{5} k_{2} k_{1} \eta C_{2}^{(0)}+\frac{1}{30} k_{2} \eta^{3} k_{1} C_{2}^{(0)} \\
& -\frac{8}{5} k_{2} C_{2}^{(1)} \eta+\frac{2}{3} k_{2} C_{2}^{(2)} \eta^{3}+\frac{4}{5} k_{2}^{2} C_{2}^{(1)} \eta-\frac{2}{15} \eta^{3} k_{2}^{2} C_{2}^{(1)} \\
& +\frac{1}{60} \eta^{3} k_{2}^{3} C_{2}^{(0)}-\frac{1}{3} \eta^{5} k_{2} C_{2}^{(2)}-\frac{1}{40} \eta^{5} k_{2} C_{2}^{(0)}+\frac{1}{60} \eta^{5} k_{1} C_{2}^{(0)} \\
& \left.+\frac{3}{10} \eta k_{2}^{2} C_{2}^{(0)}-\frac{2}{15} \eta^{5} k_{2} C_{2}^{(1)}+\frac{1}{15} \eta^{5} k_{1} C_{2}^{(1)}+\frac{1}{40} \eta^{5} k_{2}^{2} C_{2}^{(0)}+\frac{1}{15} \eta^{5} C_{2}^{(1)}+\frac{1}{3} \eta^{5} C_{2}^{(2)}+2 C_{2}^{(3)} \eta^{5}\right) e^{\left(-1 / 4 \eta^{2}\right)} \\
& -\frac{1}{120} \eta^{5} k_{2}^{2} C_{2}^{(0)}-\frac{1}{120} \eta^{5} k_{2}^{3} C_{2}^{(0)}+\frac{1}{15} \eta^{5} k_{2}^{2} C_{2}^{(1)}-\frac{1}{60} \eta^{5} k_{2} k_{1} C_{2}^{(0)} \\
& +\frac{1}{120}{ }^{(0)} \\
& \\
& \\
&
\end{aligned}
$$




$$
\begin{aligned}
+\sqrt{\pi}( & -8 k_{2} C_{2}^{(1)}+k_{1} C_{2}^{(0)}+4 C_{2}^{(1)}-40 C_{2}^{(2)}-30 C_{2}^{(2)} \eta^{2} \\
& +C_{2}^{(3)}\left(\eta^{6}+30 \eta^{4}+180 \eta^{2}+120\right)+\frac{1}{240} \eta^{6} C_{2}^{(0)}+\frac{1}{30} \eta^{6} C_{2}^{(1)} \\
& -\frac{1}{15} \eta^{6} k_{2} C_{2}^{(1)}+\frac{1}{120} \eta^{6} k_{1} C_{2}^{(0)}-\frac{1}{80} \eta^{6} k_{2} C_{2}^{(0)}+\frac{1}{80} \eta^{6} k_{2}^{2} C_{2}^{(0)} \\
& -\frac{1}{240} \eta^{6} k_{2}^{3} C_{2}^{(0)}+\frac{1}{30} \eta^{6} k_{2}^{2} C_{2}^{(1)}+\frac{1}{30} \eta^{6} k_{1} C_{2}^{(1)}-\frac{1}{6} \eta^{6} k_{2} C_{2}^{(2)} \\
& -\frac{1}{120} \eta^{6} k_{2} k_{1} C_{2}^{(0)}+\frac{1}{6} \eta^{6} C_{2}^{(2)}+40 k_{2} C_{2}^{(2)}+4 k_{2}^{2} C_{2}^{(1)} \\
& \left.-k_{2} k_{1} C_{2}^{(0)}-8 k_{1} C_{2}^{(1)}-6 k_{1} C_{2}^{(1)} \eta^{2}+30 k_{2} C_{2}^{(2)} \eta^{2}\right) \operatorname{erfc}\left(-\frac{1}{2} \eta\right)
\end{aligned}
$$

The condition at expiration tells us that $C_{1}^{(3)}=0$.

\section{REFERENCES}

[1] F. Black and M. Scholes, The pricing of options and corporate liabilities, J. Political Economy 81 (1973), 637-659.

[2] J. Cox, S. Ross, and M. Rubinstein, Option pricing: a simplified approach, J. Fin. Econ. 7 (1979), 229-264.

[3] R. Geske, A note on an analytical valuation formula for unprotected American call options on stocks with known dividends, J. Financial Econ. 7 (1979), 375-380.

[4] R. Geske and H. E. Johnson, The American put valued analytically, J. Finance 39 (1984), 1511-1524.

[5] S. D. Jacka, Optimal stopping and the American put, Mathematical Finance 1 (1991), 1-14.

[6] R. Roll, An analytical formula for unprotected American call options on stocks with known dividend, J. Financial Economics 5 (1977), 251-258.

[7] R. E. Whaley, On the valuation of American call options on stocks with known dividends, J. Financial Economics 9 (1981), 207-211.

[8] P. Wilmott, J. Dewynne, and S. Howison, Option Pricing: Mathematical Models and Computation, Financial Press, Oxford, 1995. Zbl 844.90011.

GHADA Alobaidi: Department of APPlied Mathematics, University of Western ONTARIO, LONDON, ONTARIO, CANADA N6A 5B7

E-mail address: a1obaidi@math . uregina.ca

Roland MAllier: Department of Applied Mathematics, University of Western ONTARIO, LONDON, ONTARIO, CANADA N6A 5B7

E-mail address: ma11 i er@uwo.ca 


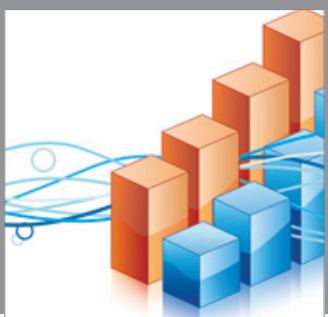

Advances in

Operations Research

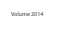

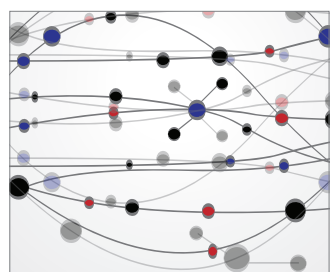

\section{The Scientific} World Journal
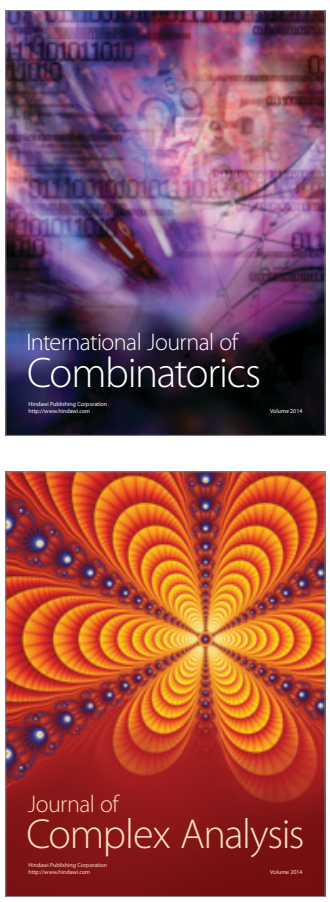

International Journal of

Mathematics and

Mathematical

Sciences
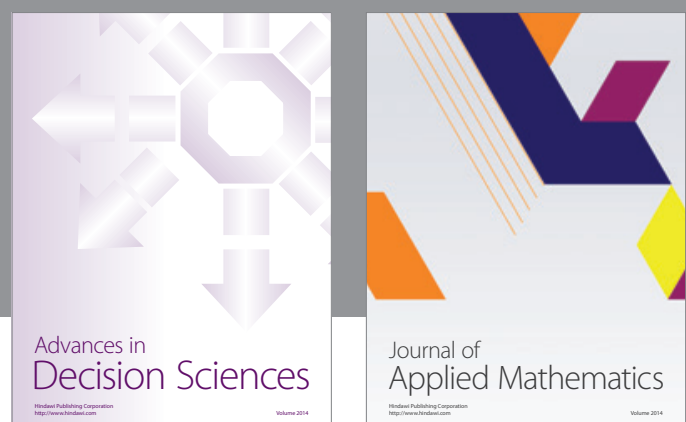

Journal of

Applied Mathematics
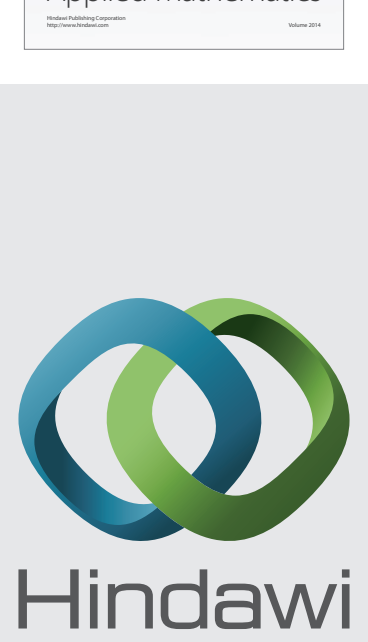

Submit your manuscripts at http://www.hindawi.com
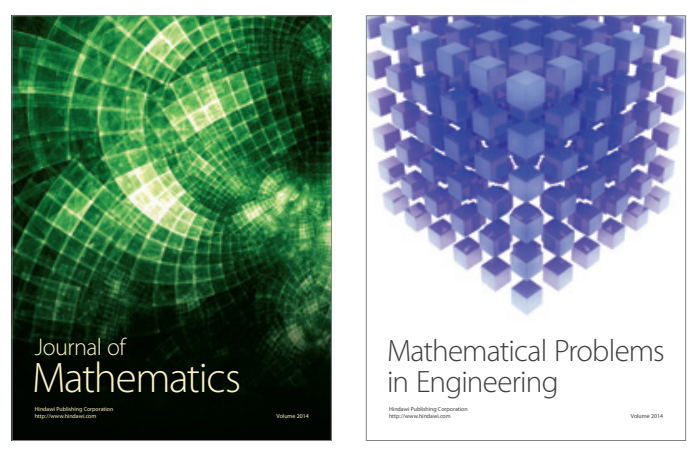

Mathematical Problems in Engineering
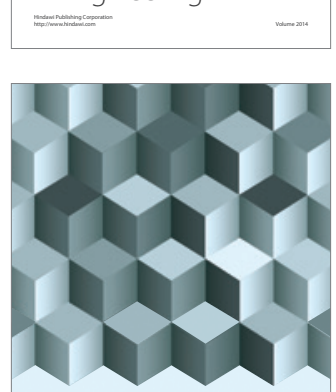

Journal of

Function Spaces
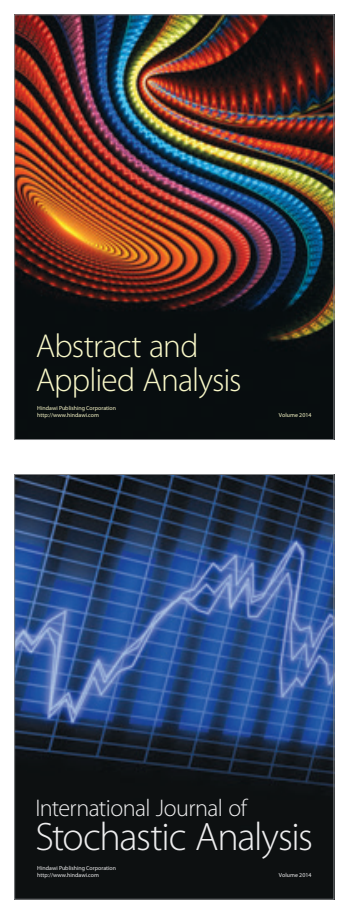

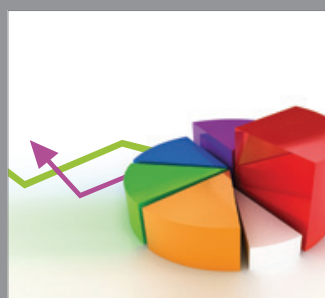

ournal of

Probability and Statistics

Promensencen
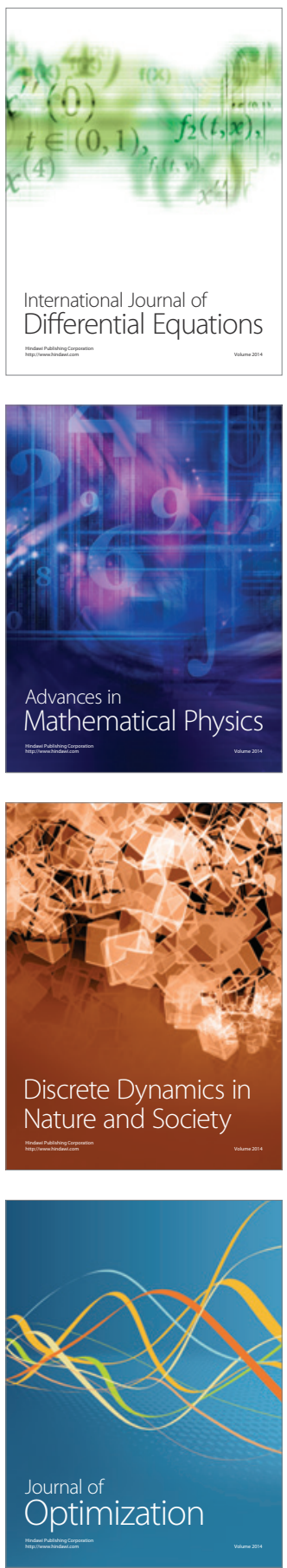